\title{
LETTER TO THE EDITOR Killer whales differentiating in geographic sympatry facilitated by divergent behavioural traditions
}

\author{
Heredity (2016) 117, 481-482; doi:10.1038/hdy.2016.112; published online 2 November 2016
}

Foote and Morin (2016) reanalyse data published in our recent RADseq studies (Moura et al., 2014a, 2015) to address questions about the likelihood of differentiation in sympatry among killer whale populations in the North Pacific. However, they describe a demic version of sympatric differentiation, requiring reproductive isolation to evolve by 'ecologically driven disruptive selection' from a background of panmixia. As they point out, questions have been raised about the potential for maintaining linkage between loci associated with ecotype and reproductive isolation, though there are some convincing putative examples of sympatric speciation by this mechanism (for example, Gavrilets et al., 2007). However, we emphasise the potential role of spatial/temporal segregation in the process, as have various authors (for example, Mallet et al., 2009). We have consistently described a process for killer whales whereby the 'social facilitation of prey location and capture' (Hoelzel et al., 2007) leads resource specialists to differential spatial and temporal habitat use, even while occupying overlapping geographic ranges, and suggested that this promotes assortative mating and differentiation by both genetic drift and selection (for example, Hoelzel et al., 2007; Moura et al., 2014a, 2015).

We agree that the dichotomous idea of allopatric vs sympatric differentiation is likely too simple, and instead consider a continuum that better reflects the real world. At the same time, there is an important distinction. Foote and Morin (2016) propose that the appearance of distinct ecotypes in the North Pacific has been seeded by admixture with populations evolving in allopatry. We instead suggest that although there may be gene flow among populations in different oceans (and we have provided evidence for such a connection between the Pacific and Atlantic in Pilot et al. (2009) and between the Pacific and Southern Oceans in Moura et al. (2014a), ecological and social factors operating within the same ocean could be sufficient to explain differentiation there. It is also apparent that similar ecotype differences (for example, separate populations of marine mammal and fish predators) occur in different oceans (see de Bruyn et al., 2013), and so requiring inter-oceanic admixture to promote this seems less parsimonious than the alternative of independent events driven by a similar mechanism (for example, resource specialisation) at each location.

Foote and Morin (2016) used the full single nucleotide polymorphisms (SNPs) data set from our population genomics paper (Moura et al., 2014a) to generate a subsample of $\sim 1300$ SNPs based on various filters. We address a number of the same issues in our phylogenomic paper (Moura et al., 2015) such as removing outliers under selection (including from the GATA4 gene), and filtering out mapping artefacts (with a different genotype calling strategy used for the final data set than in Moura et al. (2014a)). We also evaluated potential bias from concatenation by partitioning for GC content
(Romiguier et al., 2013) and using the CAT-GTR model for nucleotides (Lartillot et al., 2009). In contrast to our consensus analysis, Foote and Morin (2016) mainly considered the collective inference from a subset of selected single SNP trees. However, in a species so depauperate of diversity across the genome (see Moura et al., 2014b) each SNP has very little power to resolve the tree, and therefore the potential for stochastic inference is high. In their consensus tree, they find a similar topology to our tree (suggesting that alternative filtering did not affect the broader inference), but some nodes are less well resolved, which may reflect lower power. They investigate these poorly resolved relationships in some detail using the $\mathrm{f} 4$ statistic to distinguish between admixture and incomplete lineage sorting. However, each bootstrapped comparison is based on just five SNPs, so power is low and significance is based on $z$-scores, which assume normal distributions, unlikely for these datasets. Foote and Morin (2016) emphasise the importance of the North Atlantic population in support of their conclusions, however, our $\mathrm{ABC}$ modelling analyses that included the North Atlantic (Hoelzel and Moura, 2015) supported the topology presented in our nuclear consensus phylogeny (Moura et al., 2015).

Even so, Foote and Morin (2016) arrive at some conclusions that we not only agree with, but have reported on using different data sets from earlier studies. For example, they suggest gene flow between transient and resident populations in the North Pacific (consistent with our data from Hoelzel et al. (2007) and Moura et al. (2014a)). We note that the broader implications from the reticulate gene flow we have each described may be inconsistent with earlier proposals for multiple killer whale species (for example, Morin et al., 2010), though this can also occur among established species. Further, neither our nuclear tree nor the consensus phylogeny generated in Foote and Morin (2016) support the same topology or inference as the mtDNA tree (Foote et al., 2011; Moura et al., 2015; Hoelzel and Moura, 2015), suggesting that the topology of the single-gene tree represented by mtDNA is unlikely to represent the true species history.

Although we maintain that additional mechanisms associated with spatial and temporal isolations are likely important, there is in fact evidence for disruptive selection promoting differences between killer whale ecotypes. This has been reported in two studies, one based on RADseq data (Moura et al., 2014a), and the other on re-sequencing (Foote et al., 2016). There are limitations to each, with the RAD data based on relatively few loci, and the re-sequencing based on very low coverage for most genomes (1-2X) and small sample sizes. However, in spite of these limitations, the different approaches converge on some of the same putative loci under selection. This reinforces the idea that disruptive selection could be contributing to the differentiation of ecological specialists in geographic sympatry, even if it is not the sole mechanism. 


\section{CONFLICT OF INTEREST}

The authors declare no conflict of interest.

AR Hoelzel $^{1}$ and AE Moura ${ }^{2}$

${ }^{1}$ Department of Biosciences, Durham University, Durham, UK and ${ }^{2}$ School of Life Sciences, University of Lincoln, Lincoln, UK E-mail:a.r.hoelzel@dur.ac.uk

de Bruyn PJN, Tosh CA, Terauds A (2013). Killer whale ecotypes: is there a global model? Biol Rev 88: 62-80.

Foote AD, Morin PA (2016). Genome-wide SNP data suggest complex ancestry of sympatric North Pacific killer whale ecotypes. Heredity.

Foote AD, Morin PA, Durban JW, Willerslev E, Orlando L, Gilbert MTP (2011). Out of the Pacific and back again: insights into the matrilineal history of Pacific killer whale ecotypes. PLoS One 6: e24980.

Foote AD, Vijay N, Ávila-Arcos M, Baird RW, Durban JW, Fumagalli M et al. (2016). Genome-culture coevolution promotes rapid divergence of killer whale ecotypes. Nat Commun 7: 11693.

Gavrilets S, Vose A, Barluenga M, Salzburger W, Meyer A (2007). Case studies and mathematical models of ecological speciation. 1. Cichlids in a crater lake. Mol Ecol 16: 2893-2909.
Hoelzel AR, Hey J, Dahlheim ME, Nicholson C, Burkanov V, Black N (2007). Evolution of population structure in a highly social top predator, the Killer Whale. Mol Biol Evol 24: 1407-1415.

Hoelzel AR, Moura AE (2015). Resource specialisation and the divergence of killer whale populations. Heredity 115: 93-95.

Lartillot N, Lepage T, Blanquart S (2009). PhyloBayes 3: a Bayesian software package for phylogenetic reconstruction and molecular dating. Bioinformatics 25: 2286-2288.

Mallet J, Meyer A, Nosil P, Feder JL (2009). Space, sympatry and speciation. J Evol Biol 22: 2332-2341.

Morin PA, Archer FI, Foote AD, Vilstrup J, Allen EE, Wade P et al. (2010). Complete mitochondrial genome phylogeographic analysis of killer whales (Orcinus orca) indicates multiple species. Genome Res 20: 908-916.

Moura AE, Kenny JG, Chaudhuri R, Hughes MA, Welch A, Reisinger RR et al. (2014a). Population genomics of the killer whale indicates ecotype evolution in sympatry involving both selection and drift. Mol Ecol 23: 5179-5192.

Moura AE, Janse van Rensburg C, Pilot M, Tehrani A, Best PB, Thornton M et al. (2014b). Killer whale nuclear genome and mtDNA reveal widespread population bottleneck during the Last Glacial Maximum. Mol Biol Evol 31: 1121-1131.

Moura AE, Kenny JG, Chaudhuri R, Hughes MA, Reisinger RR, de Bruyn PJN et al. (2015). Phylogenomics of the killer whale indicates ecotype divergence in sympatry. Heredity 114: 48-55.

Pilot M, Dahlheim ME, Hoelzel AR (2009). Social cohesion among kin, gene flow without dispersal and the evolution of population genetic structure in the killer whale (Orcinus orca). J Evol Biol 23: 20-31.

Romiguier J, Ranwez V, Delsuc F, Galtier N, Douzery EJP (2013). Less is more in mammalian phylogenomics: AT-rich genes minimize tree conflicts and unravel the root of placental mammals. Mol Biol Evol 30: 2134-2144. 